\title{
Quantitative analysis of vascular endothelial growth factor receptors 1 and 2 in colorectal cancer
}

\author{
TORBEN FRØSTRUP HANSEN, KAREN-LISE GARM SPINDLER，KAREN AXELGAARD LORENTZEN, \\ DORTE AALUND OLSEN, RIKKE FREDSLUND ANDERSEN, JAN LINDEBJERG, \\ IVAN BRANDSLUND and ANDERS JAKOBSEN
}

Danish Colorectal Cancer Group South, University of Southern Denmark, Vejle Hospital, 7100 Vejle, Denmark

Received February 24, 2009; Accepted May 13, 2009

DOI: 10.3892/mmr_00000173

\begin{abstract}
Vascular endothelial growth factor A (VEGF-A) is a key regulator of angiogenesis that binds to the receptors VEGFR-1 and VEGFR-2. It is well known that VEGF-A levels are increased in colorectal cancer (CRC) tissue compared to normal colorectal tissue, but little is known regarding the expression of the VEGFR-1 and -2 receptors. The aim of the present study was to perform a quantitative analysis of VEGFR-1 and -2 at the mRNA and protein level in tumour and normal colorectal tissue from CRC patients. Tissues were collected from 110 patients who underwent surgical resection for CRC. Receptor status was analysed at the gene expression level by real time RT-PCR using $\beta$-2-microglobulin and $\beta$-actin as reference genes. Protein analysis was performed using the ELISA technique. Gene expression and protein concentrations of VEGFR-1 and -2 were significantly increased in CRC tissue compared to normal colorectal tissue $\left(\mathrm{P}<10^{-6}\right.$ for both receptors). An association between the gene expression of VEGFR-2 in CRC tissue and disease stage was detected $(\mathrm{P}=0.055)$. Significant correlations were also found between the gene expression of VEGFR-1 and -2 in CRC tissue and normal colorectal tissue $\left(\mathrm{P}<10^{-4}\right)$. The gene expression and protein concentrations of VEGFR-1 and -2 were increased in CRC tissue compared to normal colorectal tissue, indicating that both receptors are important in CRC. The clinical implications of these findings require further investigation.
\end{abstract}

\section{Introduction}

Malignant tumours have the ability to induce the formation of new capillaries from pre-existing blood vessels. This is necessary for sustained tumour growth (1). The vascular endothelial

Correspondence to: Torben Frøstrup Hansen, Department of Oncology, Vejle Hospital, Kabbeltoft 25, 7100 Vejle, Denmark

E-mail: torben.hansen@slb.regionsyddanmark.dk; torbenhansen01@hotmail.coms

Key words: colorectal neoplasm, receptors, vascular endothelial growth factor growth factor (VEGF) system is essential to angiogenesis, and targeting this system has shown promising results $(2,3)$. However, because of the toxicity and price of such treatment, reliable predictive markers are urgently needed, and a deeper understanding of the biology of the system is required.

The VEGF family includes several ligands, of which VEGF-A is the most important. VEGF-A binds to two specific receptors, VEGFR-1 (Flt-1) and VEGFR-2 (KDR or Flk-1). These receptors are mainly expressed on endothelial cells, though they are also found on a wide range of other cells, including cancer cells. VEGFR-1 and -2 are transmembraneous tyrosine kinase receptors; binding of the ligand leads to the dimerization of the receptors, and the activation of their intracellular tyrosine kinase domains. This results in the phosphorylation and activation of several downstream signalling pathways, and eventually the migration, proliferation and survival of endothelial cells and the formation of new capillaries $(4,5)$.

It has been demonstrated in several studies that VEGF-A gene expression and protein concentrations are increased in colorectal cancer (CRC) compared to normal colorectal tissue (6-9), and that VEGF-A may be a prognostic marker for the disease (10). Recently, VEGF-A gene expression and protein concentration was demonstrated to have possible predictive value regarding CRC response to chemotherapy and radiotherapy (11-14).

In contrast, little is known regarding the biological differences between tumour and normal tissue in terms of the VEGF receptors and their possible clinical relevance. The issue is of considerable interest, as an elucidation of the receptors may serve as the basis for further development of targeted therapies.

The aim of the present study was to perform a quantitative analysis of VEGFR-1 and -2 at the mRNA and protein level in tumour and normal tissue from CRC patients. To the best of our knowledge, this is the first report on the quantitative analysis of these receptors at the protein level in CRC patients.

\section{Materials and methods}

Patients. The study included 110 patients with CRC undergoing surgical resection of histologically confirmed adenocarcinomas of the colon or rectum at the Department of Surgery, Vejle Hospital, Denmark. Patients who had received preoperative 
Table I. Primers and probes for mRNA analysis.

\begin{tabular}{lll} 
Gene & & Primer and probe sequences \\
\hline VEGFR-1 & Forward primer: & CCT CTG ATG GTG ATT GTT GAA TAC TG \\
& Reverse primer: & TGC TGC ATC CTT GTT GAG AAA \\
& Probe FAM/MGB: & CAA GAG CAA ACG TGA CTT A \\
VEGFR-2 & Forward primer: & GGC TTT ACT ATT CCC AGC TAC ATG A \\
& Reverse primer: & AAC CAC ATC ATA AAT CCT ATA CCC TAC A \\
& Probe FAM/MGB: & TAT GCT GGC ATG GTC T
\end{tabular}

chemoradiation of rectal cancer were excluded. Pre-treatment examinations included a chest X-ray and a US or CT scan of the abdomen. Post-operatively, the tumours were histologically classified according to the TNM system. The study was approved by the The Danish Scientific Ethical Committee of Southern Denmark according to Danish law, and informed consent was obtained from all patients enrolled in the study.

Tissue sampling. Immediately after surgery, the resected bowel segment was transferred to the Department of Clinical Pathology. Tumour and normal mucosa samples were collected by a dedicated pathologist. Normal samples were removed as far away from the tumour as possible. Samples for RNA analysis were placed in RNAlater (Qiagen, Mainz, Germany) for further processing and stored at $-20^{\circ} \mathrm{C}$. Samples for protein analysis were fresh frozen and stored at $-80^{\circ} \mathrm{C}$. All samples were stored within 30 min of surgical removal.

Tumour tissue is a mixture of tumour and stroma cells, thus representing the tumour and its microenvironment. Based on pathological examination, it was estimated that the tumour tissue used in the present study consisted of at least $80 \%$ tumour cells.

RNA isolation and cDNA synthesis. Tissue samples stabilised in RNAlater were homogenised using the FASTPrep system (MP Biomedical, Solon, OH, USA). Total RNA was isolated using the RNeasy mini kit (Qiagen, MD, USA). Laboratory procedures were performed in accordance with the manufacturer's instructions. RNA quantification was performed using Spectrophotometry (Eppendorf, Hamburg, Germany). cDNA was synthesised using random hexamer primers and an M-MLV RT kit from Invitrogen following the manufacturer's instructions.

VEGFR mRNA analysis. The choice of reference genes was initially validated in 7 samples of normal tissue and 8 samples of tumour tissue. Six different reference genes were tested: $\beta$-2-microglobulin (B2M), succinate dehydrogenase complex (SDHA), ATP synthase (ATP5B), $\beta$-actin (ACTB), ubiquitin $C$ $(U B C)$ and glyceraldehyde-3-phosphate dehydrogenase $(G A P D H)$. Results were analysed using Normfinder (www.mdl. $\mathrm{dk}) . B 2 M$ showed the least variation between the two tissues, and $A C T B$ the second least. Further analysis showed an improvement using the two reference genes; therefore, the combination of $B 2 M$ and $A C T B$ was selected. Normalization was performed using the geometrical mean of the two refer- ence genes. The assays used were: Human B2M Endogenous Control (FAM / MGB Probe, Non-Primer Limited), Part Number 4333766F, and Human ACTB Endogenous Control (FAM / MGB Probe, Non-Primer Limited), Part Number 4333762F. Pre-manufactured assays were used (Applied Biosystems, Foster City, CA, USA).

mRNA analysis was performed using the ABI PRISM 7900 HT fast real-time PCR system (Applied Biosystems) according to standard procedures. All tests were run in triplicate. Standards and controls were analysed along with the samples. Standards used in the VEGFR-1 analysis consisted of an RNA mixture from ten different cancer cell lines from qPCR Human Reference Total RNA (HRTR) (Stratagene, La Jolla, CA, USA). RNA from Human Dermal Microvascular Endothelial Cells (CADMECs) (Cell Applications Inc., USA) was used for the VEGFR-2 analysis. Two different standards were necessary because the gene expression of VEGFR-2 was very low in the HRTR RNA, as was that of VEGFR-1 in the CADMEC RNA. The standard curves were generated on the basis of eight different dilutions of the standards. Positive controls consisting of a known amount of RNA and negative controls were included at the cDNA synthesis step and served as controls for cDNA synthesis. As the control for PCR analysis, pooled cDNA from tissue samples added to the analyses at the PCR step was used.

Primers and probes were designed using Primer Express version 3.0 (Applied Biosystems). Tests of the primers and probes regarding the recognition of genomic DNA showed no gene amplification of genomic DNA. PCR products were analysed using gel electrophoresis. The VEGFR-1 and -2 products were 96 and 132-bp long, respectively. Only the expected PCR products were detected. The assay did not detect the soluble counterpart of VEGFR-1, sVEGFR-1. Primer and probe sequences are listed in Table I.

Extraction of proteins. Tissue samples (10-50 mg), were homogenised using the ultra-turrax system (Ika, Germany) at $4^{\circ} \mathrm{C}$, with 10 volume (w/v) buffer (20 mM Tris-HCl, $150 \mathrm{mM}$ $\mathrm{NaCl}, \mathrm{pH} 7.4,1 \mathrm{mM}$ EDTA, $10 \%$ glycerol and protease inhibitor cocktail) (cat. no. P8340; Sigma-Aldrich, USA). Triton X-100 was added after homogenisation at a final sample concentration of $1 \%(\mathrm{v} / \mathrm{v})$ for the stabilisation of membrane-bound proteins. The Triton X-100 was only used under the protein extraction process, and hence did not affect the binding capacities of the standards or the controls during enzyme-linked immunosorbent assay (ELISA) analysis. For ELISA, lysates were mixed 
Table II. Clinicopathological characteristics of the patients.

\begin{tabular}{lc}
\hline Parameter & $\begin{array}{l}\text { No. }(\%) \\
(\mathrm{n}=110)\end{array}$ \\
\hline Gender & \\
Male & $54(49)$ \\
Female & $56(51)$ \\
Tumour location & \\
Colon & $72(65)$ \\
Rectum & $38(35)$ \\
pT category & \\
1 & $0(0)$ \\
2 & $13(12)$ \\
3 & $76(69)$ \\
4 & $21(19)$ \\
pN category & \\
0 & $60(55)$ \\
1 & $20(18)$ \\
2 & $30(27)$ \\
M category & \\
0 & $89(81)$ \\
1 & $21(19)$ \\
Stage & \\
II & $12(11)$ \\
\hline & $46(42)$ \\
III & $21(19)$ \\
\hline
\end{tabular}

and incubated for $30 \mathrm{~min}$ at $4{ }^{\circ} \mathrm{C}$, followed by centrifugation at $16000 \mathrm{x} g$ for $10 \mathrm{~min}$. After the recovery of the supernatants, protein concentration was determined using the bicinchorinic acid protein assay (Pierce, USA).

VEGFR protein analysis. Quantikine ELISA kits (cat. nos. DVR100B and DVR200; R\&D Systems, Minneapolis, MN, USA) were used to quantify VEGFR-1 and -2 in CRC and normal tissue. Controls were supplied by R\&D Systems. The ELISA assay employs the quantitative sandwich enzyme immunoassay technique. Analysis was performed according to the manufacturer's protocol. Optical density was determined using a Novo Biolabs kinetic microplate reader set to $450 \mathrm{~nm}$ (Molecular Devices). Data were analysed using Multicalc 2000 software.

The protein concentrations presented are expressed in $\mathrm{pg} / \mathrm{mg}$ of total protein. Tissue samples, standards and controls were assayed in duplicate, and the average was recorded. The total coefficients of variation on three levels for VEGFR1 were: low, $12.4 \%$; medium, $12.8 \%$; high, $11.3 \%$, and for VEGFR2: low, $11.3 \%$; medium, $10.1 \%$; high, $8.3 \%$.

Statistical analysis. Median values were compared using Wilcoxon's signed-rank test. Linear regression analysis was used to describe the relationship between receptor mRNA levels and protein levels in tumours and normal colorectal tissue. All statistical calculations were carried out using NCSS statistical software (Kaysville, UT, USA). P-values $<0.05$ were considered significant, and all tests were two sided.

\section{Results}

Patient characteristics. The clinicopathological characteristics of the 110 patients are listed in Table II. The median age was 72 (range 41-91) years. In $2 / 3$ of the cases, the tumour was located in the colon, and in $1 / 3$ it was in the rectum. Approximately $90 \%$ of the patients had T3 or T4 tumours, and fewer than half had lymph node metastasis.

VEGFR mRNA levels. CRC tissue and corresponding normal colorectal tissue samples from 87 patients were available for mRNA analysis using RT-PCR. Table III shows the differences between the median gene expression in the CRC and normal colorectal tissue for VEGFR-1 and -2. The differences were highly significant $\left(\mathrm{P}<10^{-6}\right)$ for both receptors.

Table IV shows differences in the distribution of clinicopathological characteristics according to the gene expression levels of both receptors with respect to age, gender, and tumour stage and location. Low gene expression of VEGFR-2 in $\mathrm{CRC}$ tissue was more common in patients with lower-stage disease than in patients with higher stages. The gene expression levels of VEGFR-1 were significantly lower in the rectal cancer patients compared to the colon cancer patients in normal tissue and tumour tissue. A similar trend was seen

Table III. Median gene expression and protein concentrations of VEGFR-1 and VEGFR-2 in CRC and normal tissue.

\begin{tabular}{|c|c|c|c|c|}
\hline & \multicolumn{2}{|c|}{ Gene expression } & \multicolumn{2}{|c|}{ Protein concentration (pg/mg) } \\
\hline & Median $(95 \% \mathrm{CI})$ & P-value & Median $(95 \% \mathrm{CI})$ & P-value \\
\hline VEGFR-1 & & $\mathrm{P}<10^{-6}$ & & $\mathrm{P}<10^{-6}$ \\
\hline Normal tissue & $2.07(1.84-2.37)$ & & $684(596-769)$ & \\
\hline CRC tissue & $4.67(4.00-5.52)$ & & $2534(2131-2789)$ & \\
\hline VEGFR-2 & & $\mathrm{P}<10^{-6}$ & & $\mathrm{P}<10^{-6}$ \\
\hline Normal tissue & $0.16(0.14-0.18)$ & & $78(66-89)$ & \\
\hline CRC tissue & $0.35(0.28-0.43)$ & & $127(118-148)$ & \\
\hline
\end{tabular}


Table IV. VEGFR-1 and VEGFR-2 gene expression according to age, gender, tumour location and stage.

\begin{tabular}{|c|c|c|c|c|c|c|c|c|}
\hline & \multicolumn{4}{|c|}{ VEGFR-1 gene expression } & \multicolumn{4}{|c|}{ VEGFR-2 gene expression } \\
\hline & \multicolumn{2}{|c|}{ Normal tissue } & \multicolumn{2}{|c|}{ Tumour tissue } & \multicolumn{2}{|c|}{ Normal tissue } & \multicolumn{2}{|c|}{ Tumour tissue } \\
\hline & Median $(95 \% \mathrm{CI})$ & P-value & Median $(95 \% \mathrm{CI})$ & P-value & Median $(95 \% \mathrm{CI})$ & P-value & Median (95\% CI) & P-value \\
\hline Age (years) & & 0.76 & & 0.48 & & 0.88 & & 0.57 \\
\hline$<72$ & $1.96(1.75-2.50)$ & & $4.57(3.54-5.74)$ & & $0.17(0.14-0.20)$ & & $0.36(0.28-0.54)$ & \\
\hline$>72$ & $2.16(1.85-2.90)$ & & $4.73(4.00-5.81)$ & & $0.16(0.14-0.18)$ & & $0.34(0.27-0.43)$ & \\
\hline Gender & & 0.020 & & 0.15 & & 0.72 & & 0.61 \\
\hline Male & $1.84(1.60-2.07)$ & & $4.37(3.45-5.52)$ & & $0.16(0.14-0.19)$ & & $0.44(0.27-0.48)$ & \\
\hline Female & $2.37(2.06-3.09)$ & & $5.22(4.04-6.04)$ & & $0.16(0.14-0.19)$ & & $0.32(0.27-0.41)$ & \\
\hline Location & & 0.005 & & 0.043 & & 0.059 & & 0.89 \\
\hline Colon & $2.19(1.96-2.89)$ & & $5.22(4.48-5.81)$ & & $0.18(0.14-0.20)$ & & $0.35(0.27-0.44)$ & \\
\hline Rectum & $1.51(1.31-2.31)$ & & $3.74(2.19-5.64)$ & & $0.15(0.09-0.17)$ & & $0.35(0.28-0.62)$ & \\
\hline Stage & & 0.27 & & 0.18 & & 0.32 & & 0.055 \\
\hline $\mathrm{I}+\mathrm{II}$ & $2.06(1.84-2.97)$ & & $4.46(3.67-5.22)$ & & $0.17(0.14-0.21)$ & & $0.28(0.25-0.35)$ & \\
\hline III+IIV & $2.08(1.64-2.31)$ & & $5.33(4.04-6.87)$ & & $0.16(0.14-0.18)$ & & $0.44(0.33-0.54)$ & \\
\hline
\end{tabular}

regarding the gene expression of VEGFR-2 in normal colorectal tissue.

VEGFR protein levels. CRC tissue and corresponding normal colorectal tissue samples from 109 patients were available for protein analysis by ELISA. Table III shows the differences between median protein concentrations in CRC and normal colorectal tissue for VEGFR-1 and -2. The differences were highly significant $\left(\mathrm{P}<10^{-6}\right)$ for both receptors.

According to the protein concentrations of the receptors, no differences in the distribution of clinicopathological characteristics with respect to age, gender, or tumour stage and location were found, with the exception of VEGFR-2 protein concentration in normal tissue, for which a lower protein

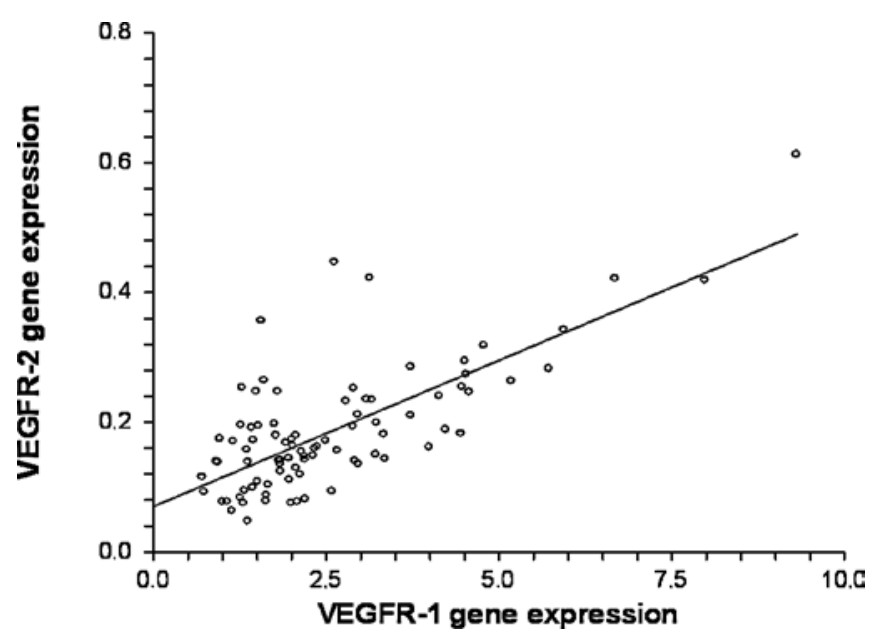

Figure 1. Linear regression plot showing VEGFR-1 and -2 gene expression in normal colorectal tissue. Vertical, gene expression of VEGF-2 in normal colorectal tissue. Horizontal, gene expression of VEGFR-1 in normal colorectal tissue. The correlation was significant $\left(\mathrm{r}=0.73\right.$ and $\left.\mathrm{P}<10^{-4}\right)$. concentration was detected in rectal cancer patients compared to colon cancer patients ( $\mathrm{P}=0.0003$; data not shown).

Correlations between the receptors. A significant correlation was found between the gene expression and protein levels of VEGFR-1 in CRC tissue $\left(\mathrm{r}=0.53, \mathrm{P}<10^{-4}\right)$. The same cannot be said for VEGFR-2 $(\mathrm{r}=0.20, \mathrm{P}=0.072)$. There were no correlations between the gene expression and protein levels of VEGFR-1 and -2 in normal tissue. A significant correlation was found between the gene expression of VEGFR-1 and -2 in $\mathrm{CRC}$ tissue $\left(\mathrm{r}=0.57, \mathrm{P}<10^{-4}\right)$ and normal colorectal tissue, $\left(\mathrm{r}=0.73, \mathrm{P}<10^{-4}\right)$ (Fig. 1).

\section{Discussion}

The survival of patients with mCRC has increased due to the incorporation of drugs targeting the VEGF system into the chemotherapy regimens currently in use. However, much remains to be learnt regarding the biology of the VEGF system in order for this treatment modality to be improved. There is an increasing interest in the receptors of the VEGF system, but the potential clinical implication of receptor status remains more or less unknown. Furthermore, quantitative analyses at the protein level of VEGFR-1 and -2 in CRC or normal colorectal tissue have not been previously performed. As a result, we decided to analyse VEGFR-1 and -2 at the gene expression and protein levels in CRC and normal colorectal tissue.

In the present study, analysis at the mRNA level showed that the gene expression of both receptors was higher in CRC tissue than in normal colorectal tissue. This is in agreement with a study by Hanrahan et al ( $71 \mathrm{CRC}$ patients, $>80 \%$ in stages I-II and none in stage IV; only 20 samples representing normal mucosa) in which the authors found the gene expression level of VEGFR-1 and -2 to be higher in CRC tissue than in normal colorectal tissue (15). In a study by André et al (78 CRC patients, stages I-IV; primarily patients $<75$ years) also showed 
a higher gene expression of VEGFR-1 in CRC tissue compared to normal colonic tissue. However, no difference was detected in relation to VEGFR-2 gene expression (6). The lack of differences in the gene expression levels of VEGFR-2 in CRC tissue compared to normal colorectal tissue have been shown in other studies, for example those of Georges et al (40 CRC patients, stages I-IV, $>60 \%$ stages III-IV): Di et al (48 CRC patients, stages I-IV) and Parr et al (28 CRC patients, no information on clinical pathological characteristics) $(7,16,17)$. In comparing the different studies, differences in the patient sample, especially the number of patients included, must be noted, and the results should therefore be interpreted with caution. Furthermore, differences in the methods of analysis may have affected the findings, and must be taken into account. Lastly, tumour heterogeneity cannot be excluded.

The correlation between the low disease stage and the low gene expression of VEGFR-2, but not VEGFR-1, in CRC tissue might indicate a difference in the influence of the two receptors at different stages of disease. In the present study, it appeared that VEGFR-2 gene expression was up-regulated during the advanced stages of disease. A similar trend was found in a study by Di et al, where the VEGFR-2 gene expression carcinoma/ mucosa ratio increased from 0.4 in stage I to 1.4 in stage IV $(\mathrm{P}=0.11)$ (16). Contrary to our findings, Hanrahan et al observed a significant increase in VEGFR-1 gene expression in stage III patients compared to stage I+II patients, and found no differences in the gene expression of VEGFR-2 according to stage (15); however, the number of patients sampled was rather small. It is worth noting that none of these three studies showed increased gene expression of any of the receptors during the lower stages of the disease. It would therefore appear that the gene expression of these two receptors is increased in the higher stages of CRC compared to the lower stages. Increases in tumour dependency on angiogenesis and in disease stage seem reasonable in light of current knowledge.

The present results also show that protein concentrations of VEGFR-1 and -2 were increased in CRC tissue compared to normal colorectal tissue. The protein concentration of VEGFR-1 in CRC tissue was increased by a factor of $3.6 \mathrm{com}-$ pared to concentrations in normal colorectal tissue, while the corresponding factor for VEGFR-2 was 1.6. This is supported by the results of testing at the gene expression level. In the literature, we found no studies measuring VEGFR-1 or VEGFR-2 protein concentrations in CRC and normal colorectal tissue using the quantitative ELISA technique. A few studies using semi-quantitative techniques, such as immunohistochemistry, found indications of higher VEGFR-2 protein expression in CRC compared to normal colorectal tissue $(18,19)$, but a direct comparison with our study is of course not possible due to the substantial differences in the techniques employed.

The higher concentrations of the receptors in CRC tissue compared to normal colorectal tissue found underline the higher metabolic demands and angiogenetic activity known to exist in malignant tissue. Furthermore, the findings indicate that both receptors are of clinical importance in CRC.

To some extent, lower gene expression levels and protein concentrations of VEGFR-1 and -2 were found in rectal cancer patients compared to colon cancer patients in both normal and tumour tissue. This implies the existence of differences in angiogenetic activity and microenvironmental conditions in relation to the VEGF system between colonic and rectal tissues. However, these results are only hypothesis generating, and should be confirmed in further studies.

For VEGFR-1, a significant correlation between gene expression and protein concentration was detected in CRC tissue, while for VEGFR-2 only a trend was observed. A possible explanation for this finding could be a difference between the two genes in terms of the rate of activation and deactivation. Significant correlations between the gene expression of the receptors were detected in both tissues. A previous study by Azuma et al showed a similar correlation between the gene expression of VEGFR-1 and -2 in tumour tissue from 36 patients with metastatic CRC (20). The significant correlations between the gene expression of the receptors in both types of tissue in our study indicates that the gene expression of these two receptors is regulated at least in part by the same mechanisms, and that the isolated up-regulation of one receptor is uncommon.

This study has the standard limitations related to retrospective studies. The relatively small number of patients is also a limitation, but to date this study is the biggest reported to conduct the quantitative analysis of VEGFR-1 and VEGFR-2. Many of the results should be considered as hypothesis generating rather than facts, and validation in another cohort of patients is necessary.

In conclusion, the results indicate that the gene expression and protein concentration of VEGFR-1 and -2 are higher in $\mathrm{CRC}$ tissue than in normal colorectal tissue. The gene expression of VEGFR-2 in CRC tissue seems to be correlated with the stage of disease. Furthermore, correlations between the gene expression of both receptors were detected in the tissues, suggesting a uniform regulation. The possible clinical implications of these findings require further investigation.

\section{Acknowledgements}

We are very thankful for technical assistance provided by Lone Frischknecht, Lone Hartmann Hansen, Sara Egsgaard and Birgit Roed Sørensen, and for proofreading by Karin Larsen. The study was supported by the Cancer Foundation.

\section{References}

1. Folkman $\mathrm{J}$ : What is the evidence that tumors are angiogenesis dependent? J Natl Cancer Inst 82: 4-6, 1990.

2. Hurwitz H, Fehrenbacher L, Novotny W, Cartwright T, Hainsworth J, Heim W, Berlin J, Baron A, Griffing S, Holmgren E, Ferrara N, Fyfe G, Rogers B, Ross R and Kabbinavar F: Bevacizumab plus irinotecan, fluorouracil and leucovorin for metastatic colorectal cancer. N Engl J Med 350: 2335-2342, 2004.

3. Kim KJ, Li B, Winer J, Armanini M, Gillett N, Phillips HS and Ferrara N: Inhibition of vascular endothelial growth factorinduced angiogenesis suppresses tumour growth in vivo. Nature 362: 841-844, 1993.

4. Ferrara N, Gerber HP and LeCouter J: The biology of VEGF and its receptors. Nat Med 9: 669-676, 2003.

5. Takahashi $\mathrm{H}$ and Shibuya $\mathrm{M}$ : The vascular endothelial growth factor (VEGF)/VEGF receptor system and its role under physiological and pathological conditions. Clin Sci (Lond) 109: 227-241, 2005.

6. Andre T, Kotelevets L, Vaillant JC, Coudray AM, Weber L, Prevot S, Parc R, Gespach C and Chastre E: Vegf, Vegf-B, Vegf-C and their receptors KDR, FLT-1 and FLT-4 during the neoplastic progression of human colonic mucosa. Int J Cancer 86: 174-181, 2000.

7. George ML, Tutton MG, Janssen F, Arnaout A, Abulafi AM, Eccles SA and Swift RI: VEGF-A, VEGF-C and VEGF-D in colorectal cancer progression. Neoplasia 3: 420-427, 2001. 
8. Ferroni P, Spila A, Martini F, D'Alessandro R, Mariotti S, Del MG, Graziano P, Buonomo O, Guadagni F and Roselli M: Prognostic value of vascular endothelial growth factor tumor tissue content of colorectal cancer. Oncology 69: 145-153, 2005.

9. Altomare DF, Rotelli MT, Pentimone A, Rossiello MR, MartinelliE, Guglielmi A, De FM, Marino F, Memeo V, Colucci M and Semeraro N: Tissue factor and vascular endothelial growth factor expression in colorectal cancer: relation with cancer recurrence. Colorectal Dis 9: 133-138, 2007.

10. Des GG, Uzzan B, Nicolas P, Cucherat M, Morere JF, Benamouzig R, Breau JL and Perret GY: Microvessel density and VEGF expression are prognostic factors in colorectal cancer. Meta-analysis of the literature. Br J Cancer 94: 1823-1832, 2006.

11. Vallbohmer D, Zhang W, Gordon M, Yang DY, Yun J, Press OA Rhodes KE, Sherrod AE, Iqbal S, Danenberg KD, Groshen S and Lenz HJ: Molecular determinants of cetuximab efficacy. J Clin Oncol 23: 3536-3544, 2005.

12. Vincenzi B, Santini D, Russo A, Gavasci M, Battistoni F Dicuonzo G, Rocci L, Rosaria VM, Gebbia N and Tonini G: Circulating VEGF reduction, response and outcome in advanced colorectal cancer patients treated with cetuximab plus irinotecan. Pharmacogenomics 8: 319-327, 2007.

13. Zlobec I, Vuong T, Compton CC, Lugli A, Michel RP, Hayashi S and Jass JR: Combined analysis of VEGF and EGFR predicts complete tumour response in rectal cancer treated with preoperative radiotherapy. Br J Cancer 98: 450-456, 2008.

14. Zlobec I, Steele R and Compton CC: VEGF as a predictive marker of rectal tumor response to preoperative radiotherapy. Cancer 104: 2517-2521, 2005.
15. Hanrahan V, Currie MJ, Gunningham SP, Morrin HR, Scott PA, Robinson BA and Fox SB: The angiogenic switch for vascular endothelial growth factor (VEGF)-A, VEGF-B, VEGF-C and VEGF-D in the adenoma-carcinoma sequence during colorectal cancer progression. J Pathol 200: 183-194.

16. Di FF, Alvarado C, Majdan A, Gologan A, Voda L, Mitmaker E, Beitel LK, Gordon PH and Trifiro M: Underexpression of mineralocorticoid receptor in colorectal carcinomas and association with VEGFR-2 overexpression. J Gastrointest Surg 11: 1521-1528, 2007.

17. Parr C and Jiang WG: Quantitative analysis of lymphangiogenic markers in human colorectal cancer. Int J Oncol 23: 533-539, 2003.

18. Duff SE, Jeziorska M, Rosa DD, Kumar S, Haboubi N, Sherlock D, O'Dwyer ST and Jayson GC: Vascular endothelial growth factors and receptors in colorectal cancer: implications for anti-angiogenic therapy. Eur J Cancer 42: 112-117, 2005.

19. Giatromanolaki A, Koukourakis MI, Sivridis E, Chlouverakis G, Vourvouhaki E, Turley H, Harris AL and Gatter KC: Activated VEGFR2/KDR pathway in tumour cells and tumour associated vessels of colorectal cancer. Eur J Clin Invest 37: 878-886, 2007.

20. Azuma M, Shi M, Danenberg KD, Gardner H, Barrett C, Jacques CJ, Sherod A, Iqbal S, El-Khoueiry A, Yang D, Zhang W, Danenberg PV and Lenz HJ: Serum lactate dehydrogenase levels and glycolysis significantly correlate with tumor VEGFA and VEGFR expression in metastatic CRC patients. Pharmacogenomics 8: 1705-1713, 2007. 